\title{
OGLE-2017-BLG-0482Lb: A Microlensing Super-Earth Orbiting a Low-mass Host Star
}

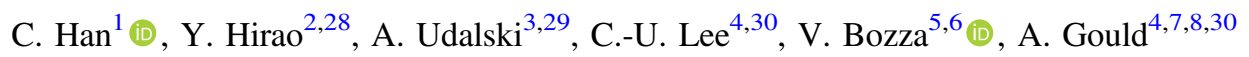

and

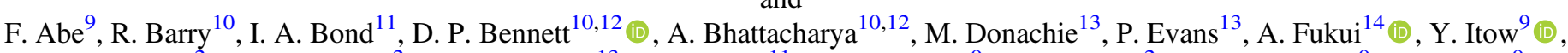
K. Kawasaki ${ }^{2}$, N. Koshimoto ${ }^{2}$ (1), M. C. A. Li $^{13}$, C. H. Ling ${ }^{11}$, Y. Matsubara ${ }^{9}$, S. Miyazaki ${ }^{2}$ (1), H. Munakata ${ }^{9}$, Y. Muraki ${ }^{9}$, M. Nagakane ${ }^{2}$, K. Ohnishi ${ }^{15}$, C. Ranc ${ }^{10}$, N. Rattenbury ${ }^{13}$ (D) T. Saito ${ }^{16}$, A. Sharan ${ }^{13}$, D. J. Sullivan ${ }^{17}$, T. Sumi ${ }^{2}$, D. Suzuki ${ }^{18}$ (D), P. J. Tristram ${ }^{19}$, T. Yamada $^{2}$, A. Yonehara ${ }^{20}$

(The MOA Collaboration),

P. Mróz ${ }^{3}$, R. Poleski ${ }^{3,7}$, S. Kozłowski ${ }^{3}$, I. Soszyński ${ }^{3}$, P. Pietrukowicz ${ }^{3}$ (1) J. Skowron ${ }^{3}$ (1), M. K. Szymański ${ }^{3}$, K. Ulaczyk ${ }^{3}$, M. Pawlak ${ }^{3}$, K. Rybicki ${ }^{3}$, P. Iwanek ${ }^{3}$

(The OGLE Collaboration),

and

M. D. Albrow ${ }^{21}$, S.-J. Chung ${ }^{4,22}$, K.-H. Hwang ${ }^{4}$, Y. K. Jung ${ }^{23}$, D. Kim ${ }^{1}$, W.-T. Kim ${ }^{24}$ (D) H.-W. Kim ${ }^{4}$, Y.-H. Ryu ${ }^{4}$, I.-G. Shin ${ }^{23}$, Y. Shvartzvald ${ }^{25,31}$ (D), J. C. Yee ${ }^{23}$, W. Zhu ${ }^{26}$, S.-M. Cha ${ }^{4,27}$, S.-L. Kim ${ }^{4,22}$, D.-J. Kim ${ }^{4}$, D.-J. Lee ${ }^{4}$, Y. Lee ${ }^{4,27}$, B.-G. Park ${ }^{4,22}$, and R. W. Pogge 7

(The KMTNet Collaboration)

${ }^{1}$ Department of Physics, Chungbuk National University, Cheongju 28644, Republic of Korea; cheongho@ astroph.chungbuk.ac.kr

${ }^{2}$ Department of Earth and Space Science, Graduate School of Science, Osaka University, Toyonaka, Osaka 560-0043, Japan

${ }^{3}$ Warsaw University Observatory, Al. Ujazdowskie 4, 00-478 Warszawa, Poland

${ }^{4}$ Korea Astronomy and Space Science Institute, Daejon 34055, Republic of Korea

${ }^{5}$ Dipartimento di Fisica "E. R. Caianiello", Universitá di Salerno, Via Giovanni Paolo II, I-84084 Fisciano (SA), Italy

${ }^{6}$ Istituto Nazionale di Fisica Nucleare, Sezione di Napoli, Via Cintia, I-80126 Napoli, Italy

${ }^{7}$ Department of Astronomy, Ohio State University, 140 W. 18th Avenue, Columbus, OH 43210, USA

${ }^{8}$ Max Planck Institute for Astronomy, Königstuhl 17, D-69117 Heidelberg, Germany

${ }^{9}$ Institute for Space-Earth Environmental Research, Nagoya University, 464-8601 Nagoya, Japan

${ }^{10}$ Code 667, NASA Goddard Space Flight Center, Greenbelt, MD 20771, USA

${ }^{11}$ Institute of Natural and Mathematical Sciences, Massey University, Auckland 0745, New Zealand

${ }^{12}$ Deptartment of Physics, University of Notre Dame, 225 Nieuwland Science Hall, Notre Dame, IN 46556, USA

${ }^{13}$ Dept. of Physics, University of Auckland, Private Bag 92019, Auckland, New Zealand

${ }^{14}$ Okayama Astrophysical Observatory, National Astronomical Observatory of Japan, Asakuchi, 719-0232 Okayama, Japan ${ }^{15}$ Nagano National College of Technology, 381-8550 Nagano, Japan

${ }^{16}$ Tokyo Metroplitan College of Industrial Technology, 116-8523 Tokyo, Japan

17 School of Chemical and Physical Sciences, Victoria University, Wellington, New Zealand

${ }^{18}$ Institute of Space and Astronautical Science, Japan Aerospace Exploration Agency, Kanagawa 252-5210, Japan

${ }^{19}$ Mt. John University Observatory, P.O. Box 56, Lake Tekapo 8770, New Zealand

${ }^{20}$ Department of Physics, Faculty of Science, Kyoto Sangyo University, 603-8555 Kyoto, Japan

${ }^{21}$ University of Canterbury, Department of Physics and Astronomy, Private Bag 4800, Christchurch 8020, New Zealand

${ }^{22}$ Korea University of Science and Technology, 217 Gajeong-ro, Yuseong-gu, Daejeon 34113, Republic of Korea

${ }^{23}$ Harvard-Smithsonian Center for Astrophysics, 60 Garden St., Cambridge, MA, 02138, USA

${ }^{24}$ Department of Physics \& Astronomy, Seoul National University, Seoul 151-742, Republic of Korea

25 Jet Propulsion Laboratory, California Institute of Technology, 4800 Oak Grove Drive, Pasadena, CA 91109, USA

${ }^{26}$ Canadian Institute for Theoretical Astrophysics, University of Toronto, 60 St George Street, Toronto, ON M5S 3H8, Canada

${ }^{27}$ School of Space Research, Kyung Hee University, Yongin 17104, Republic of Korea

Received 2018 February 7; revised 2018 March 25; accepted 2018 March 27; published 2018 April 25

\begin{abstract}
We report the discovery of a planetary system in which a super-Earth orbits a late M-dwarf host. The planetary system was found from the analysis of the microlensing event OGLE-2017-BLG-0482, wherein the planet signal appears as a short-term anomaly to the smooth lensing light curve produced by the host. Despite its weak signal and short duration, the planetary signal was firmly detected from the dense and continuous coverage by three microlensing surveys. We find a planet/host mass ratio of $q \sim 1.4 \times 10^{-4}$. We measure the microlens parallax $\pi_{\mathrm{E}}$ from the long-term deviation in the observed lensing light curve, but the angular Einstein radius $\theta_{\mathrm{E}}$ cannot be measured because the source trajectory did not cross the planet-induced caustic. Using the measured event timescale and the microlens parallax, we find that the masses of the planet and the host are $M_{\mathrm{p}}=9.0_{-4.5}^{+9.0} M_{\oplus}$ and $M_{\text {host }}=0.20_{-0.10}^{+0.20} M_{\odot}$, respectively, and the projected separation between them is $a_{\perp}=1.8_{-0.7}^{+0.6}$ au. The estimated distance to the lens is $D_{\mathrm{L}}=5.8_{-2.1}^{+1.8} \mathrm{kpc}$. The discovery of the planetary system demonstrates that microlensing provides an important method to detect low-mass planets orbiting low-mass stars.
\end{abstract}

Key words: gravitational lensing: micro - planetary systems

\footnotetext{
${ }^{28}$ The MOA Collaboration.

${ }^{29}$ OGLE Collaboration.

30 The KMTNet Collaboration.

${ }^{31}$ NASA Postdoctoral Program Fellow.
} 


\section{Introduction}

The last two decades have witnessed a great increase of the number of known planetary systems, which is reaching 2800 as of the end of 2017 according to the Extrasolar Planet Encyclopedia (http://exoplanet.eu/catalog/). Notably, the Kepler mission using the transit method has contributed to the dramatic increase in the number of known planetary systems. However, most of the known planets belong to solar-type stars, and the number of planets orbiting low-mass stars is much smaller than that of planets orbiting solar-type stars. In particular, planets around late M-type dwarfs comprise a very small portion of known planets due to the difficulty of observing their host stars.

Planets around low-mass stars may be formed through a different process from those orbiting solar-type stars. For example, the luminosity of low-mass stars decreases substantially (by a factor of $\sim 10-100$ ) on the Hayashi track during typical planet formation timescales of 10-100 Myr, while the luminosity of solar-mass stars remains almost constant (Kennedy et al. 2006). The difference in the environment between the two types of stars would likely affect the evolution of stellar disks and the subsequent planet formation processes. See the review of Boss (1989) about the planet formation process of low-mass stars. However, the details of the planet formation process for low-mass stars are poorly known because the planet sample is too small to check proposed scenarios. As a result, our understanding of planets around low-mass stars is incomplete despite the fact that the hosts are the most common population of stars in the Galaxy.

Microlensing occurs by the gravitational field of an intermediary objects between an observer and a background star. Due to this nature, planet detections using the microlensing method do not rely on the host's luminosity but just its gravity and that of the planet, while other planet detection methods rely on the luminosity of the host. This enables one to extend microlensing planet searches to stars with very low luminosities and even substellar brown-dwarf hosts, e.g., OGLE-2012-BLG-0358Lb (Han et al. 2013). Furthermore, the method is sensitive to low-mass planets down to Earth-mass planets, e.g., OGLE-2016-BLG-1195Lb (Bond et al. 2017; Shvartzvald et al. 2017). For this reason, microlensing planets comprise $\sim 23 \%$ of the known planets with host masses $\lesssim 0.2 M_{\odot}$ and planet masses $\lesssim 10 M_{\oplus}$, although they comprise only $\sim 2 \%$ of the total planet sample.

In this work, we report the microlensing discovery of a superEarth planet orbiting a low-mass M-dwarf host. The planetary system was found from the analysis of the microlensing event OGLE-2017-BLG-0482, in which the planet revealed its presence as a short-term anomaly. Despite the short duration, the planet signal was firmly detected from the combination of three high-cadence lensing surveys.

\section{Observations and Data}

In Figure 1, we present the light curve of the lensing event OGLE-2017-BLG-0482. The event occurred on a faint star with a baseline magnitude $I \sim 20$. The source star is located toward the Galactic bulge field with equatorial coordinates (R.A., decl.) $)_{\mathrm{J} 2000}=$ $(17: 56: 11.73,-30: 31: 42.1)$, which correspond to the Galactic coordinates $(l, b)=(-0.2,-2.8)$. The amplification of the source flux induced by lensing was first noticed on 2017 April 8 $\left(\mathrm{HJD}^{\prime}=\mathrm{HJD}-2450,000 \sim 7852\right)$ by the Optical Gravitational Lensing Experiment (OGLE; Udalski et al. 2015) survey that is conducted using the $1.3 \mathrm{~m}$ Warsaw telescope at Las Campanas
Observatory in Chile. Images of the OGLE survey were taken mainly in the $I$ band, and some $V$-band images were taken for color measurement.

The event was also in the observation fields of the Microlensing Observations in Astrophysics (MOA; Bond et al. 2001; Sumi et al. 2003) survey and the Korea Microlensing Telescope Network (KMTNet; Kim et al. 2016) survey. Data of the MOA survey were taken using the $1.8 \mathrm{~m}$ telescope located at the Mt. John University Observatory in New Zealand. In the list of MOA transient events, the event is denoted by MOA-2017-BLG-209. MOA data were acquired using a customized $R$ band that has a bandwidth corresponding to roughly the sum of the standard $R$ and $I$ bands. The KMTNet data were obtained using its three globally distributed $1.6 \mathrm{~m}$ telescopes located at the Cerro Tololo Interamerican Observatory in Chile (KMTC), the South African Astronomical Observatory in South Africa (KMTS), and the Siding Spring Observatory in Australia (KMTA). KMTNet observations were conducted in the $I$ - and $V$-band filters. The KMTNet data obtained by each telescope are composed of two sets (denoted by BLG01 and BLG41) because the survey alternately covered the field with a $6^{\prime}$ offset to fill gaps between the camera chips. In the list of KMTNet microlensing candidates, it is called SAO01M0605.043904 (Kim et al. 2018a, 2018b).

After it was detected, the light curve of the event followed the smooth form of a single-mass lensing event reaching a peak magnification $A_{\max } \sim 16$ at $\mathrm{HJD}^{\prime} \sim 7874$. On May 6 UT 15:29 $\left(\mathrm{HJD}^{\prime} \sim 7880.15\right)$, the MOA group alerted the microlensing community to a possible planetary anomaly based on real-time assessment by the MOA observer with the aim of encouraging follow-up observations. Unfortunately, no follow-up observation could be conducted mainly due to the short duration of the anomaly. A day after the anomaly alert, Y. Hirao of the MOA group released a model of the anomaly based on the MOA data. According to this model, the anomaly was produced by the crossing of the source over the caustic produced by a planetary companion with a mass ratio of $q \sim 10^{-4}$. V. Bozza also released a similar model. From modeling conducted with the addition of data from the OGLE and KMTNet surveys, it was noticed that the earlier models exhibit inconsistency with the additional data, and an updated model without caustic crossing was presented by C. Han. After the anomaly, the event followed the light curve of a single-mass event and gradually returned to the baseline.

The firm detection and characterization of this weak and short planetary signal was made possible by the combination of the three high-cadence lensing surveys. In the upper panel of Figure 1, we present the zoom of the planet-induced anomaly. The anomaly lasted only for about 2 days. Furthermore, the signal is weak with a maximum deviation of $\sim 0.2$ mag relative to the single-mass lensing light curve. Nevertheless, the signal was densely and continuously covered by the survey experiments thanks to the high-cadence observations conducted using globally distributed telescopes.

Photometry of the data is conducted using softwares customized by the individual groups based on the Difference Imaging Analysis (Alard \& Lupton 1998; Woźniak 2000): Udalski (2003) for the OGLE, Bond et al. (2001) for the MOA groups, and pyDIA developed by M. Albrow for the KMTNet. For the KMTC data set, an additional photometry is conducted with DoPHOT software (Schechter et al. 1993) for the determination of the source color and the construction of a 

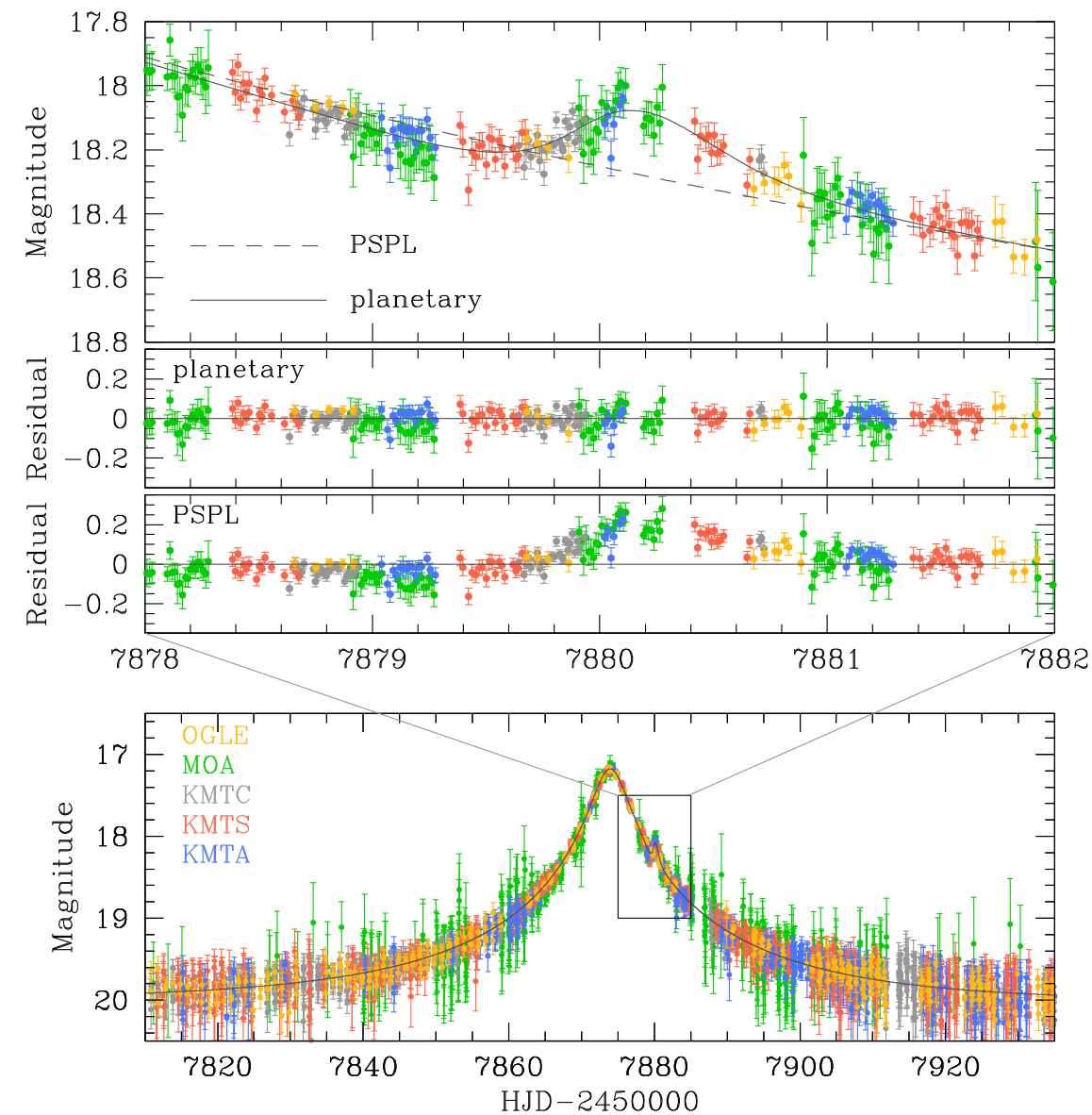

Figure 1. Light curve of the gravitational microlensing event OGLE-2017-BLG-0482. The upper panels show the zoom of the planetary anomaly region that is enclosed by a box in the bottom panel. The solid and dashed curves superposed on the data are the best-fit planetary model and the point-source point-lens (PSPL) model, respectively. The middle two panels show the residuals from the individual models.

color-magnitude diagram. For the use of heterogeneous data sets obtained using different instruments and processed using different photometry codes, error bars of the individual data sets are readjusted following the procedure described by Yee et al. (2012).

\section{Analysis}

\subsection{Planetary Analysis}

The observed light curve appears to be a typical case of a planetary lensing event in which the planetary signal is revealed as a short-term perturbation to the smooth light curve produced by the host of the planet (Mao \& Paczyński 1991; Gould \& Loeb 1992). For the basic description of the lensing light curve produced by a lens composed of two masses, one requires six parameters. Three of these parameters describe the geometry of the lens-source approach: the time of the closest lens-source separation, $t_{0}$, the lens-source separation at that time, $u_{0}$ (normalized to the angular Einstein radius $\theta_{\mathrm{E}}$ ), and the time for the source to cross the Einstein radius, $t_{\mathrm{E}}$ (Einstein timescale). The other three parameters describe the binarity of the lens: the separation between the primary $\left(M_{1}\right)$ and the companion $\left(M_{2}\right), s$ (also normalized to $\theta_{\mathrm{E}}$ ), their mass ratio, $q=M_{2} / M_{1}$, and the angle between the source trajectory and the binary axis, $\alpha$ (source trajectory angle). If the source crosses the planet-induced caustic, the perturbation is affected by finite-source effects (Bennett \& Rhie 1996). In such cases, one needs an additional parameter $\rho=\theta_{*} / \theta_{\mathrm{E}}$ (normalized source radius) to account for the light curve deviation caused by the finite-source effect. Here, $\theta_{*}$ represents the angular radius of the source star.

In cases for which planetary signals can be treated as perturbations, one can heuristically estimate the lensing parameters related to the planet, i.e., $s$ and $q$, from the location and duration of the perturbation (Gould \& Loeb 1992; Gaudi 2012). A planet induces two types of caustics: central and planetary caustics. The central caustic lies close to the primary of the lens, and thus the caustic produces perturbations near the peak of high magnification events. The planetary caustic, on the other hand, lies away from the primary lens with a separation

$$
u_{\mathrm{p}}=s-\frac{1}{s} \text {, }
$$

and thus perturbations induced by the planetary caustic can appear anywhere along the lensing light curve. The perturbation of OGLE-2017-BLG-0482 lies away from the peak and thus it is produced by a planetary caustic. The perturbation occurred when the lensing magnification was $A=\left(u^{2}+2\right) /\left[u\left(u^{2}+4\right)^{1 / 2}\right] \sim 7.5$, i.e., when the lenssource separation was $u \sim 0.135$. By substituting $u$ into $u_{\mathrm{p}}$ in Equation (1), one finds that the planet-host separation is either $s \sim 0.93$ or 1.07 , which are referred to as the close $(s<1)$ and wide-separation $(s>1)$ solutions, respectively. The caustic induced by a close planet usually produces a dip (negative deviation) in the light curve, while the caustic induced by a 


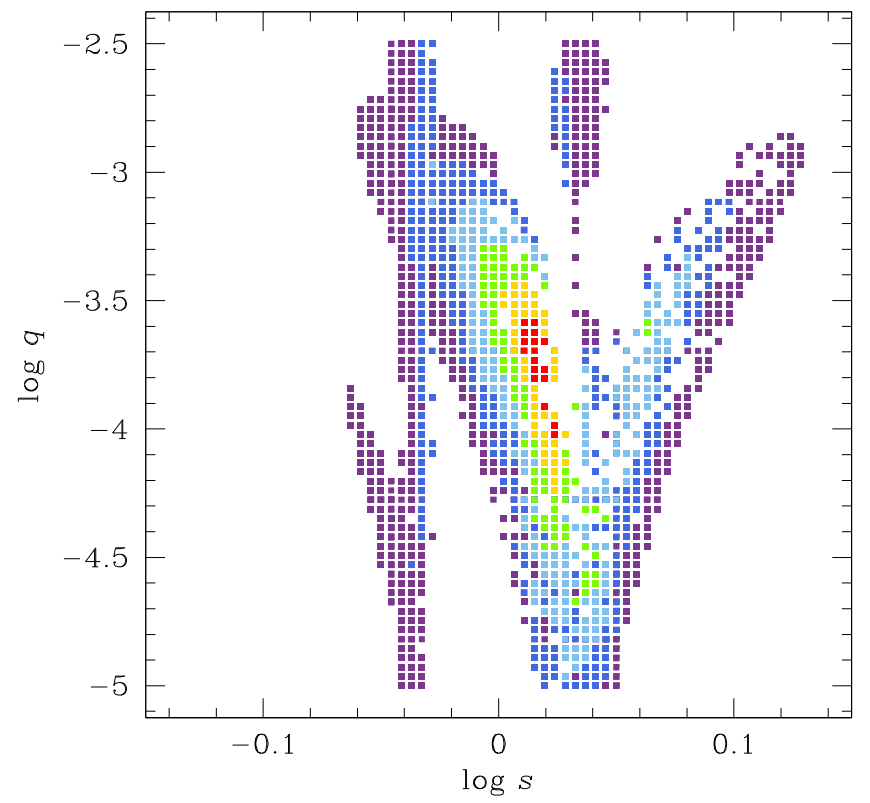

Figure 2. Distribution of $\Delta \chi^{2}$ in the $(\log s, \log q)$ plane. Locations marked in different colors represent the regions with $\Delta \chi^{2}<n^{2}$ (red), $(2 n)^{2}$ (yellow), $(3 n)^{2}$ (green), $(4 n)^{2}$ (cyan), $(5 n)^{2}$ (blue), and $(6 n)^{2}$ (purple) from the best-fit solution, where $n=5$.

wide planet always produces a bump (positive deviation). The observed bump structure of the perturbation, therefore, suggests that the planet separation is greater than $\theta_{\mathrm{E}}$, i.e., $s \sim 1.07$.

The duration of the planet-induced perturbation results from the combination of the sizes of the caustic, $\Delta \xi_{\mathrm{p}}$, and the source, $\rho$. If the source is bigger than the caustic, $\rho>\Delta \xi_{\mathrm{p}}$, the duration corresponds to the source crossing time, $\Delta t_{\mathrm{p}} \sim 2 \rho t_{\mathrm{E}}$, and thus is mostly determined by the source radius. If the source is smaller than the caustic, on the other hand, the duration is determined by the size of the caustic. In the case of OGLE2017-BLG-0482, the source is a very faint main-sequence star and thus the duration is likely to depend on the caustic size. The size of the planetary caustic is related to the separation and the mass ratio between the planet and the host by

$$
\Delta \xi_{\mathrm{p}}=\frac{4 q^{1 / 2}}{s^{2}}\left(1+\frac{1}{2 s^{2}}\right)
$$

Han (2006). As the duration of the planetary signal is $\Delta t_{\mathrm{p}} \sim$ $\Delta \xi_{\mathrm{p}} t_{\mathrm{E}}$, the mass ratio is expressed by

$$
q=\left(\frac{s^{4}}{4 s^{2}+2} \frac{\Delta t_{\mathrm{p}}}{t_{\mathrm{E}}}\right)^{2} .
$$

With $s \sim 1.07, \Delta t_{\mathrm{p}} \sim 2$ days, and $t_{\mathrm{E}} \sim 40$ days, one finds that the mass ratio is $\sim 10^{-4}$.

For the accurate determinations of the lensing parameters, we conduct numerical modeling of the observed light curve. We search for the solution of the lensing parameters in two steps. In the first step, we conduct a dense grid search over $(\log s, \log q)$ plane. At each point on this plane, we hold the two grid parameters fixed while allowing the remaining five parameters $\left(t_{0}, u_{0}, t_{\mathrm{E}}, \rho, \alpha\right)$ to vary in six Markov Chain Monte Carlo (MCMC) $\chi^{2}$ minimizations that are equally spaced around the circle in their seed values of $\alpha$. The seed values of $\left(t_{0}, u_{0}, t_{\mathrm{E}}\right)$ are taken from the point-lens fit, and we seed $\rho$ at

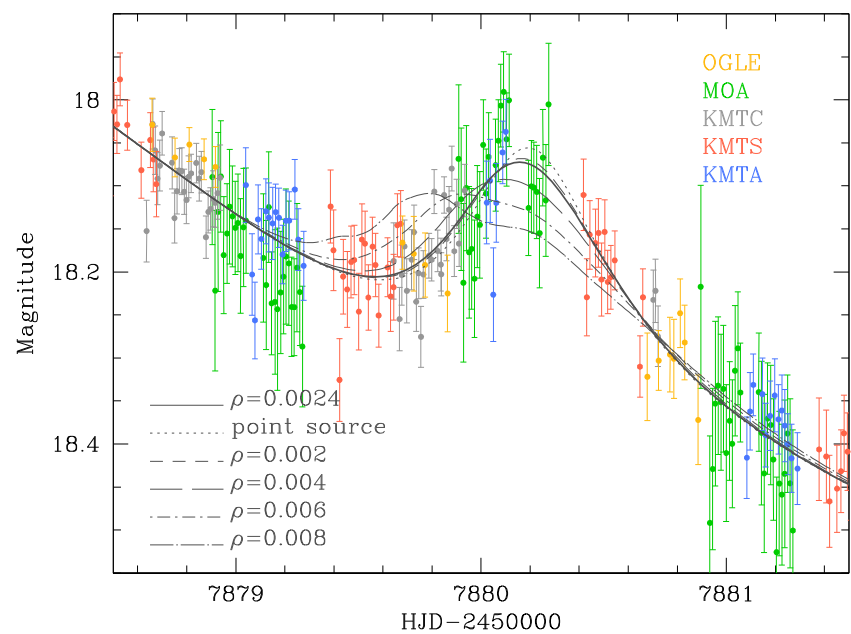

Figure 3. Model light curves expected from various values of the normalized source radius $\rho$.

$\rho=1.0 \times 10^{-3}$. From this preliminary search, we identify local $\chi^{2}$ minima on the $(\log s, \log q)$ plane. In the second step, we refine the individual local minima by allowing all parameters to vary. Figure 2 displays the $\Delta \chi^{2}$ map over the $(\log s, \log q)$ plane obtained from the grid search. It shows that there exists a unique planetary solution with the planet separation slightly greater than unity and a mass ratio of $q=(1-3) \times 10^{-4}$, which roughly matches the prediction of the heuristic analysis.

\subsection{Finite-source Effects}

When a source passes over or approaches very close to caustics, the planetary anomaly is affected by finite-source effects and the analysis of the deviation enables one to measure the normalized source radius $\rho$. In the case of OGLE-2017BLG-0482, it is found that $\rho$ cannot be measured due to the lack of finite-source effects. Measuring $\rho$ is important because the angular Einstein radius, which is needed to determine the lens mass, is estimated from $\rho$ by $\theta_{\mathrm{E}}=\theta_{*} / \rho$. The difficulty of the $\rho$ measurement is caused by the fact that the source did not cross the caustic.

Nevertheless, the source approached close to a strong cusp of the caustic around which the gradient of lensing magnification is high. In this case, one can set an upper limit on the source size. To test this possibility, we draw light curves expected from various values of $\rho$ (see Figure 3 ). From this, combined with the MCMC chain obtained from modeling, we find that the $3 \sigma$ upper limit of the normalized source size is $\rho_{\max } \sim$ 0.006 . However, a lower limit cannot be set because the best-fit model cannot be distinguished from a point-source model within $2 \sigma$.

\subsection{Binary-source Interpretation}

It is known that a subset of binary-source events can produce short-term anomalies similar to planetary perturbations and thus masquerade as planetary events (Gaudi 1998; Hwang et al. 2013, 2017). We, therefore, check the possibility of the interpretation in which the perturbation is produced by a source companion. The lensing magnification of a binary-source event corresponds to the flux-weighted mean of the magnifications 


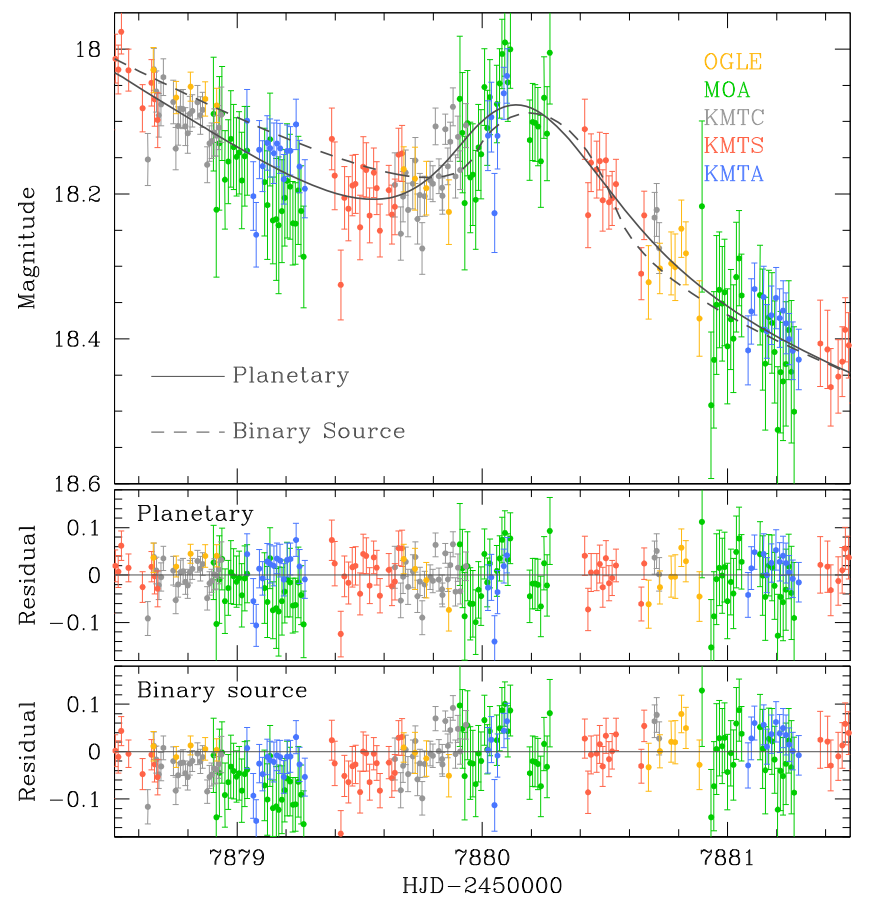

Figure 4. Comparison of the planetary (solid curve) and binary-source (dashed curve) models. The two lower panels show the residuals from the individual models.

associated with the individual source stars, $A_{1}$ and $A_{2}$, i.e.,

$$
A=\frac{A_{1} F_{1}+A_{2} F_{2}}{F_{1}+F_{2}}=\frac{A_{1}+q_{F} A_{2}}{1+q_{F}} .
$$

Here, $q_{F}=F_{2} / F_{1}$ represents the ratio between the unmagnified fluxes of the individual source stars (Han \& Jeong 1998). We conduct modeling of the observed data with the binary-source interpretation. For a binary-source event mimicking a planetary event, the flux ratio is usually very small, and the faint source approaches close to the lens. In this case, lensing magnifications during the perturbation can be affected by finite-source effects. $\mathrm{We}$, therefore, consider finite-source effects in the modeling.

In Figure 4, we compare the fits of the planetary and binarysource models in the neighborhood of the anomaly. The best-fit binary-source model yields a flux ratio $q_{F, I} \sim 0.005$. One finds that the binary-source model yields an unsatisfactory description of the region before the major anomaly, i.e., $7878.5 \lesssim \mathrm{HJD}^{\prime} \lesssim$ 7879.7. Numerically, we find that the binary-source model is worse than the planetary model by $\Delta \chi^{2}=175.2$. We, therefore, exclude the binary-source interpretation.

\subsection{Higher-order Effects}

To precisely describe lensing light curves, it is often necessary to consider higher-order effects. In the case of OGLE-2017-BLG0482 , the event duration of $\Delta t=2\left(1-u_{0}^{2}\right)^{1 / 2} t_{\mathrm{E}} \sim 80$ days, as measured by the time during which the source is within the Einstein ring, comprises an important portion of Earth's orbital period, i.e., 1 year. In this case, the light curve can deviate from the one expected from a rectilinear lens-source relative motion due to the orbital motion of Earth: "microlens-parallax" effect (Gould 1992). Similarly, the orbital motion of the lens can also induce deviation in the lensing light curve: "lens-orbital" effect (Albrow et al. 2000).
Consideration of the higher-order effects requires additional parameters in lensing modeling. To account for the microlensparallax effect, one needs two parameters: $\pi_{\mathrm{E}, N}$ and $\pi_{\mathrm{E}, E}$. These parameters denote the north and east components of the microlens-parallax vector, $\pi_{\mathrm{E}}$, projected onto the sky along the north and east equatorial coordinates, respectively. The magnitude of the microlens-parallax vector is

$$
\pi_{\mathrm{E}}=\left(\pi_{\mathrm{E}, N}^{2}+\pi_{\mathrm{E}, E}^{2}\right)^{1 / 2}=\frac{\pi_{\mathrm{rel}}}{\theta_{\mathrm{E}}},
$$

where $\pi_{\mathrm{rel}}=\mathrm{au}\left(D_{\mathrm{L}}^{-1}-D_{\mathrm{S}}^{-1}\right)$ is the lens-source relative parallax, and $D_{\mathrm{L}}$ and $D_{\mathrm{S}}$ represent the distances to the lens and source, respectively. The direction of $\pi_{\mathrm{E}}$ corresponds to the relative lens-source motion, $\boldsymbol{\mu}$. To the first-order approximation that the change rates of the binary separation and source trajectory angle are constant, the lens-orbital effect is described by two parameters, $d s / d t$ and $d \alpha / d t$, which represent the change rates of the binary separation and the source trajectory angle, respectively. The measurement of the microlens parallax is important to determine the physical parameters of the lens mass and distance because $\pi_{\mathrm{E}}$ is related to these parameters by

$$
M=\frac{\theta_{\mathrm{E}}}{\kappa \pi_{\mathrm{E}}},
$$

and

$$
D_{\mathrm{L}}=\frac{\mathrm{au}}{\pi_{\mathrm{E}} \theta_{\mathrm{E}}+\pi_{\mathrm{S}}},
$$

where $\kappa=4 G /\left(c^{2} \mathrm{au}\right)$ and $\pi_{\mathrm{S}}=\mathrm{au} / D_{\mathrm{S}}$ is the parallax of the source.

In order to check the higher-order effects, we conduct additional modeling of the observed data. From this modeling, we find that the fit substantially improves (by $\Delta \chi^{2} \sim 110$ ) with the consideration of the higher-order effects. However, it is found find that the signal of the higher-order effects varies depending on the data sets. From the inspection of the cumulative $\Delta \chi^{2}$ distributions as a function of time for the individual data sets, we find that most of the signal comes from the KMTC (by $\Delta \chi^{2} \sim 90$ ) and the KMTS (by $\Delta \chi^{2} \sim 20$ ) data sets, while the signal from the other data sets is minor. The inconsistency of the signal among different data sets is of concern because signals of the higher-order effects are subtle long-term deviations from the standard model, and thus they can be affected by the stability of photometry data. We additionally check the reality of the signal by replacing the KMTC and KMTS photometry data with new ones processed using a different software, pySIS (Albrow et al. 2000). From this, we find that the inconsistency between the KMTC +KMTS and the other data sets still persists. These results suggest the possibility that the KMTC and KMTS data are not stable enough to securely measure the higher-order parameters.

Knowing the possibility of systematics in the KMTC and KMTS data, we mainly use the OGLE data set to measure the higher-order parameters. The KMTNet survey started in 2015 season and thus the system is still under development. On the other hand, the OGLE system is very stable from its 25 -year operation since 1992. While the higher-order effect parameters are determined based on the overall shape of the lensing light curve, the planet parameters are determined by the planetary anomaly. The overall light curve was well covered by the OGLE data, but the OGLE coverage around the planetary 

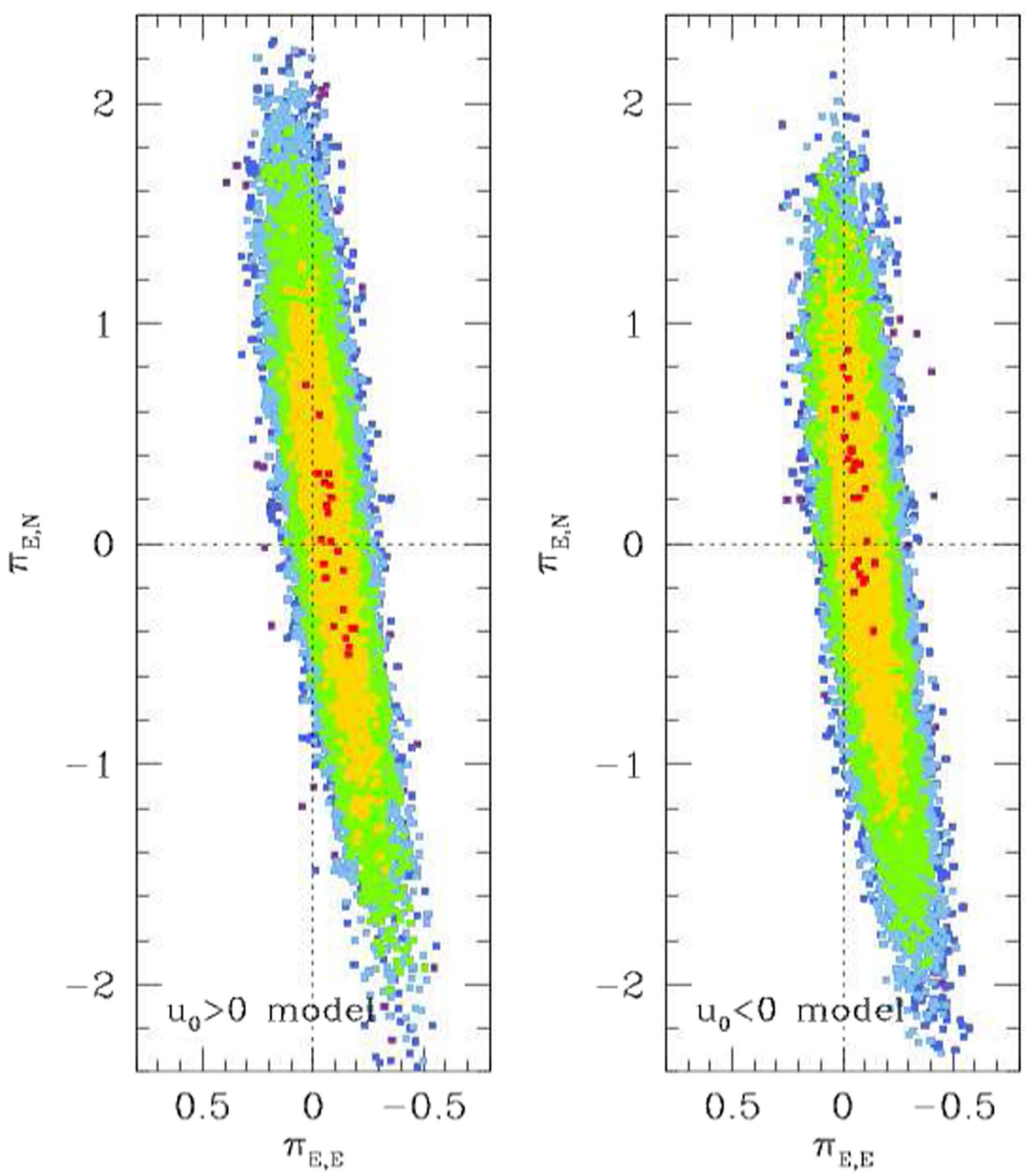

Figure 5. $\Delta \chi^{2}$ maps in the $\left(\pi_{\mathrm{E}, E}, \pi_{\mathrm{E}, N}\right)$ plane. The left and right panels show the $u_{0}>0$ and $u_{0}<0$ solutions, respectively. Color coding is same as in Figure 2 except that $n=1$. The maps are based on the truncated data set, which is explained in Section 3.4.

anomaly is poor. In the analysis, we therefore use the combination of data sets with the whole OGLE data set plus partial data sets from the other data sets. The KMTNet+MOA data used in the analysis cover the anomaly region during $7875<$ HJD $^{\prime}<7887$. We exclude MOA data outside this region as well because of the instability in the baseline.

In Figure 5, we present the $\Delta \chi^{2}$ map of MCMC chains in the $\left(\pi_{\mathrm{E}, E}, \pi_{\mathrm{E}, N}\right)$ plane obtained using the the restricted data set. In the model considering the microlens-parallax effect, it is known that there exist a pair of degenerate solutions with $u_{0}>0$ and $u_{0}<0$ due to the mirror symmetry of the source trajectories between the two degenerate solutions (Smith et al. 2003; Skowron et al. 2011), and thus we check this so-called ecliptic degeneracy. We note that the lensing parameters of the two solutions resulting from the ecliptic degeneracy are approximately in the relation $\left(u_{0}, \alpha, \pi_{\mathrm{E}, N}, d \alpha / d t\right) \leftrightarrow-\left(u_{0}, \alpha, \pi_{\mathrm{E}, N}, d \alpha / d t\right)$. From the distributions of MCMC points, we find that both $u_{0}>0$ and $u_{0}<0$ solutions result in similar values of $\pi_{\mathrm{E}}$. The measured microlensparallax parameters are $\left(\pi_{\mathrm{E}, N}, \pi_{\mathrm{E}, E}\right)=(0.19 \pm 0.75,-0.06 \pm$ $0.12)$ for the $u_{0}>0$ model and $(-0.17 \pm 0.73,-0.12 \pm 0.11)$ for the $u_{0}<0$ model. It is found that the east component of the microlens-parallax vector, $\pi_{\mathrm{E}, E}$, is well constrained but the uncertainty of the north component, $\pi_{\mathrm{E}, N}$, is considerable.
In Table 1, we list the lensing parameters of the best-fit solutions. Because the ecliptic degeneracy is severe with $\Delta \chi^{2}<1$, we present both solutions with $u_{0}>0$ and $u_{0}<0$. Also presented are the flux from the source $F_{s}$ and the blended light $F_{b}$ measured from the OGLE data. We find that the event was produced by a planetary system with $s \sim 1.07$ and $q \sim 1.4 \times 10^{-4}$.

In Figure 6, we present the lens system geometry (for the $u_{0}>0$ solution), in which the source trajectory with respect to the lens and caustic are shown. Due to the proximity of the normalized planet-host separation to unity, i.e., $s \sim 1$, the caustics form a single closed curve for which the central and planetary caustics are merged into a single caustic, i.e., resonant caustic. The planetary perturbation was produced when the source approached the strong planet-side cusp of the caustic located on the planet-host axis. As mentioned in Section 3.2, the source trajectory did not pass over the caustic and thus finite-source effects cannot be measured. We note that the uncertainties of the lens-orbital parameters $d s / d t$ and $d \alpha / d t$ are big, indicating that the lens-orbital motion is poorly constrained. Nevertheless, it is important to fit for these parameters because they can be correlated with the parallax parameters (Batista et al. 2011; Skowron et al. 2011). 

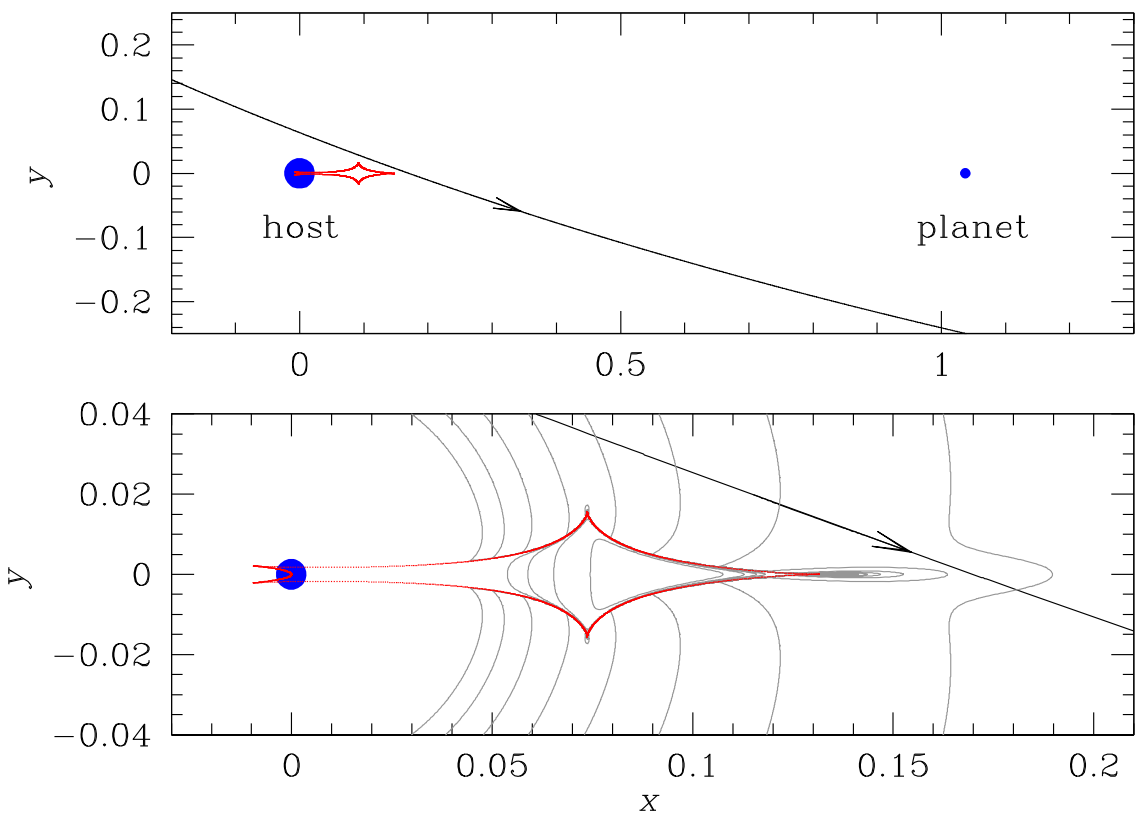

Figure 6. Geometry of the lens system. The upper panel shows the positions of both the host and planet while the lower panel shows the zoom around the caustic. The curve with an arrow represents the source trajectory. The lens components are marked by blue dots where the bigger one represents the host and the smaller one is the planet. The red cuspy closed curve is the caustic. All lengths are scaled to the angular Einstein radius corresponding to the total mass of the lens. The gray curves around the caustic represent the contours of lensing magnifications with $A=6,8,10,12,14,16,18$, and 20, respectively.

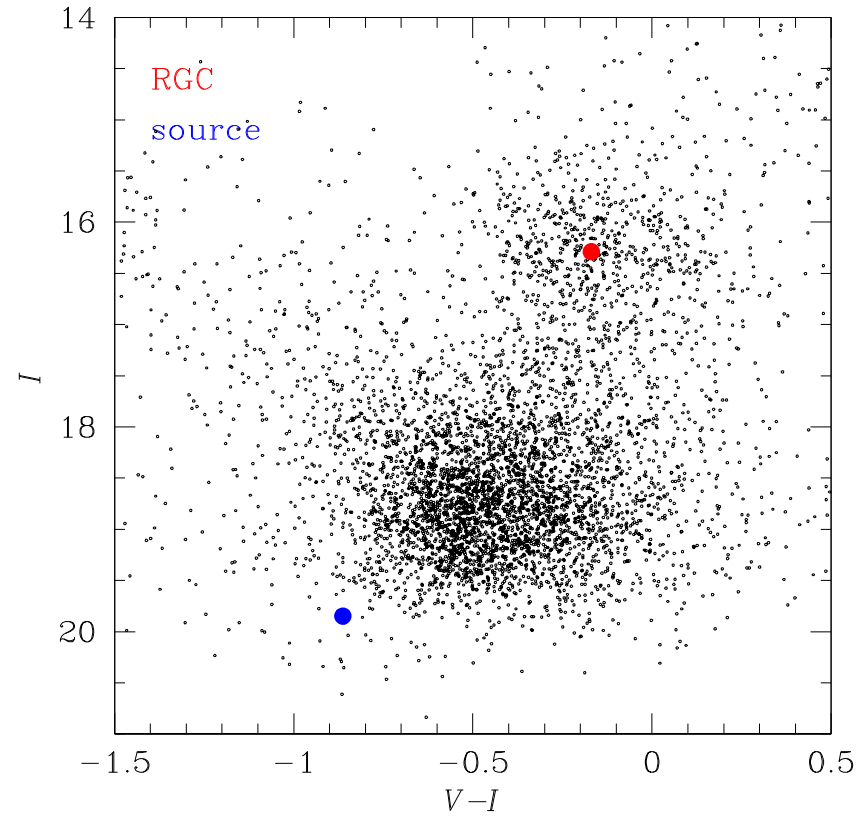

Figure 7. The locations of the source star (blue dot) and the centroid of red giant clump (RGC, red dot) in the instrumental color-magnitude diagram. The source is unusually blue $(V-I)_{0}=0.37$ relative to most microlensed sources and therefore is inferred to lie in the Galactic disk rather than the bulge.

\subsection{Source Star}

In the analysis of lensing events, characterizing the source star is important because the angular Einstein radius, which is needed to determine the lens mass and distance by Equations (6) and (7), is derived from the angular source radius by $\theta_{\mathrm{E}}=\theta_{*} / \rho$. In the case of OGLE-2017-BLG-0482, the normalized source radius $\rho$ and thus the angular Einstein radius $\theta_{\mathrm{E}}$ cannot be measured. However, the upper limit on $\rho$ leads to a lower limit on $\theta_{\mathrm{E}}$, and this lower limit may help to
Table 1

Best-fit Lensing Parameters

\begin{tabular}{lcc}
\hline \hline \multirow{2}{*}{ Parameter } & \multicolumn{2}{c}{ Value } \\
\cline { 2 - 3 } & $u_{0}>0$ & $u_{0}<0$ \\
\hline$\chi^{2}$ & 1548.4 & 1548.6 \\
$t_{0}\left(\mathrm{HJD}^{\prime}\right)$ & $7873.948 \pm 0.013$ & $7873.952 \pm 0.013$ \\
$u_{0}$ & $0.059 \pm 0.002$ & $-0.058 \pm 0.002$ \\
$t_{\mathrm{E}}($ days $)$ & $40.01 \pm 1.45$ & $40.39 \pm 1.21$ \\
$s$ & $1.07 \pm 0.01$ & $1.07 \pm 0.01$ \\
$q\left(10^{-4}\right)$ & $1.35 \pm 0.20$ & $1.41 \pm 0.30$ \\
$\alpha(\mathrm{rad})$ & $0.369 \pm 0.023$ & $-0.365 \pm 0.026$ \\
$\pi_{\mathrm{E}, N}$ & $0.19 \pm 0.75$ & $-0.17 \pm 0.73$ \\
$\pi_{\mathrm{E}, E}$ & $-0.06 \pm 0.12$ & $-0.12 \pm 0.11$ \\
$d s / d t\left(\mathrm{yr}^{-1}\right)$ & $-1.94 \pm 0.82$ & $-1.96 \pm 0.96$ \\
$d \alpha / d t\left(\mathrm{yr}^{-1}\right)$ & $-0.29 \pm 0.27$ & $0.87 \pm 0.50$ \\
$F_{s, \mathrm{OGLE}}$ & $0.124 \pm 0.006$ & $0.122 \pm 0.004$ \\
$F_{b, \mathrm{OGLE}}$ & $0.029 \pm 0.005$ & $0.031 \pm 0.004$ \\
\hline
\end{tabular}

Note. HJD $^{\prime}=$ HJD -2450000 .

constrain the physical lens parameters. Thus, determination of $\theta_{*}$ could be important depending on what limit is obtained.

We characterize the source based on its dereddened color $(V-I)_{0}$ and brightness $I_{0}$. To determine $(V-I)_{0}$ and $I_{0}$ from the instrumental color $V-I$ and brightness $I$, we apply the method of Yoo et al. (2004) using the centroid of red giant clump (RGC) as a reference. Figure 7 shows the locations of the source and the RGC centroid in the instrumental color-magnitude diagram. With the offsets in color and brightness $\Delta(V-I, I)=(-0.69,3.56)$ with respect to the RGC centroid and the known dereddened color and brightness of the RGC centroid of $(V-I, I)_{0, \mathrm{RGC}}=(1.06,14.46)$ (Bensby et al. 2013; Nataf et al. 2013), it is estimated that the dereddened 

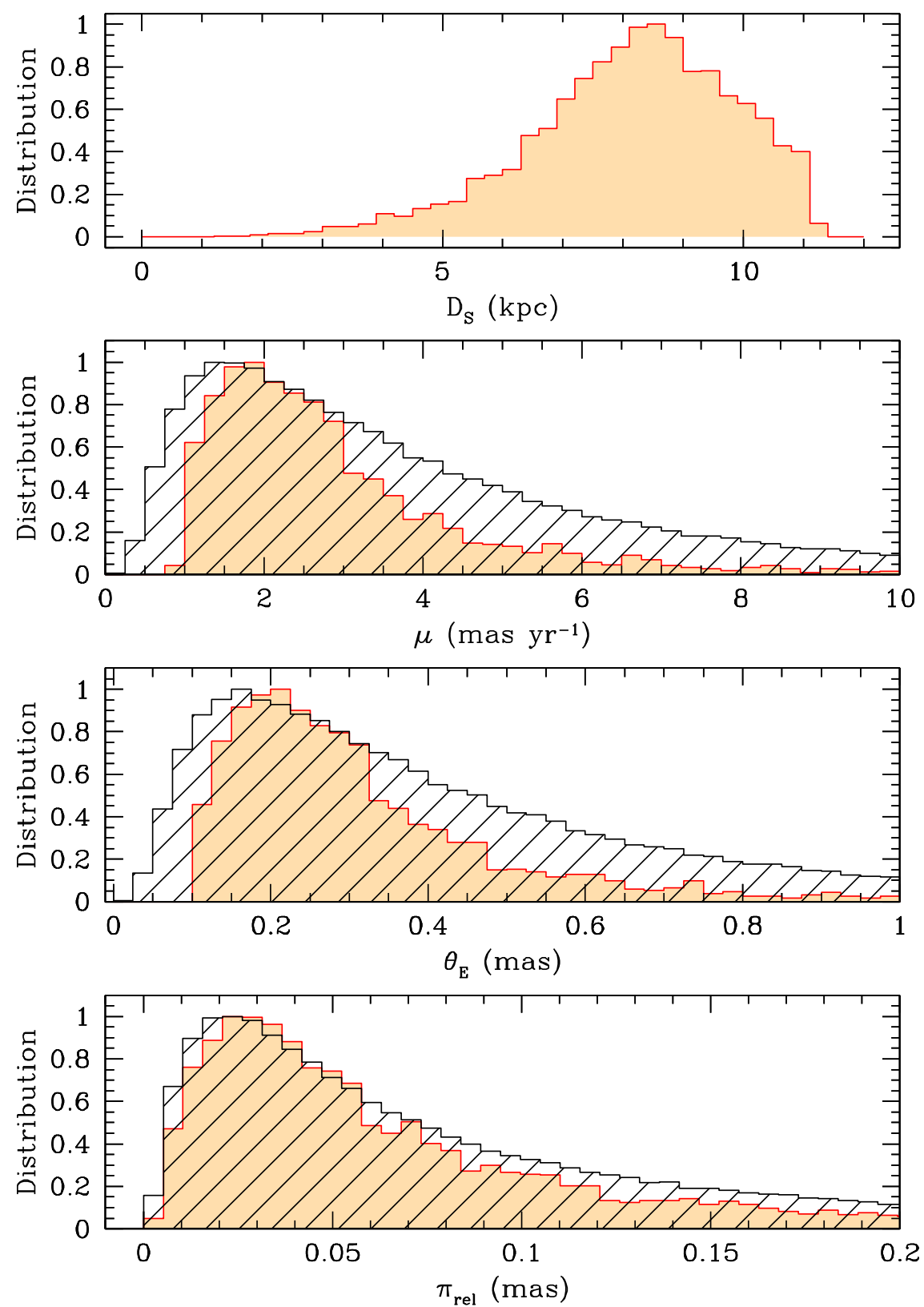

Figure 8. The distributions of the source distance $\left(D_{\mathrm{S}}\right)$, the angular Einstein radius $\theta_{\mathrm{E}}$ (middle panel), and the relative lens-source parallax $\pi_{\mathrm{rel}}=\operatorname{au}\left(D_{\mathrm{L}}^{-1}-D_{\mathrm{S}}^{-1}\right)$ produced from the Bayesian analysis using the adopted Galactic models. For $\mu, \theta_{\mathrm{E}}$, and $\pi_{\text {rel }}$, we present two sets of distributions: one with (histogram filled with a yellow shade) and the other without (histogram shaded by slanted lines) the constraint of the measured $t_{\mathrm{E}}$ and $\pi_{\mathrm{E}}$.

color and brightness of the source are $(V-I, I)_{0}=$ $(V-I, I)_{0, \mathrm{RGC}}+\Delta(V-I, I)=(0.37,18.02)$. We note that the relatively blue color indicates that the source is a mainsequence star located in the disk. Considering the faintness, the star is likely to lie behind the obscuring dust.

Once the dereddened $V-I$ color is measured, we then use the VIK color-color relations of Bessell \& Brett (1988) to convert from $V-I$ to $V-K$ and apply the color/surface-brightness relation of Kervella et al. (2004) to obtain $\theta_{*}=0.56 \mu$ as. Combined with the $3 \sigma$ upper limit we have derived on $\rho$ and the measured Einstein timescale, this implies

$$
\theta_{\mathrm{E}}=\frac{\theta_{*}}{\rho}>0.093 \mathrm{mas}
$$

and

$$
\mu=\frac{\theta_{\mathrm{E}}}{t_{\mathrm{E}}}>0.86 \operatorname{mas} \mathrm{yr}^{-1}
$$

We note that the lower limits of $\theta_{\mathrm{E}}$ and $\mu$ can be subject to additional uncertainties because of the uncertain ratio between the extinction values toward the source and the RGC centroid. The method using a color-magnitude diagram to derive $\theta_{*}$ assumes that the amount of extinction toward the source and RGC stars is same. For OGLE-2017-BLG-0482, the source is likely to be in the disk, and thus the hypothesis of the same extinction may not be valid. If the source suffers less extinction than RGC stars, it would be actually fainter and redder than if it were at the same distance as the RGC stars. Therefore, the 

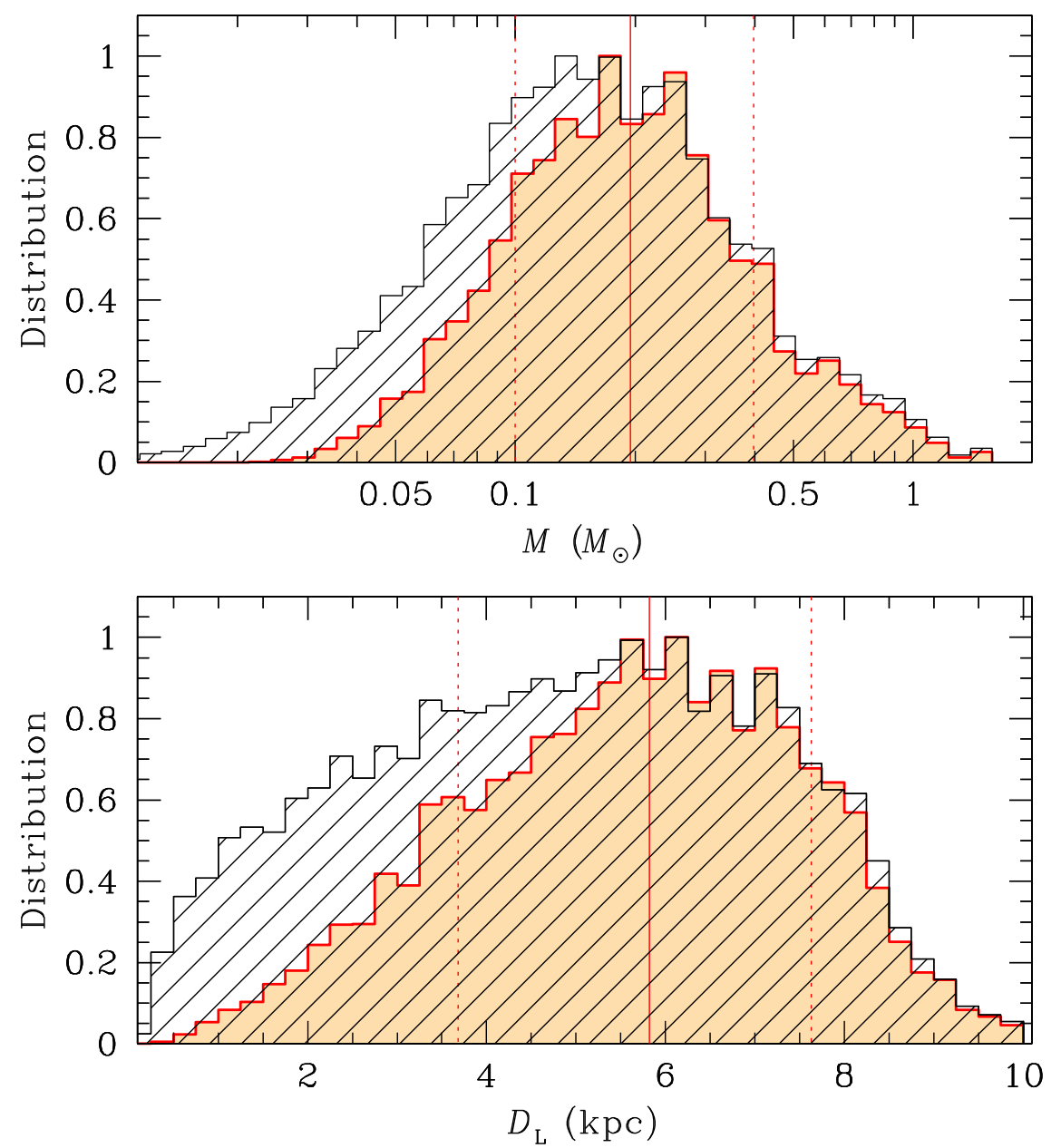

Figure 9. Distributions of the mass (upper panel) and the distance to the lens (lower panel) obtained from the Bayesian analysis. The solid vertical line represents the median, and the dotted lines represent the $1 \sigma$ range of the distribution. In each panel, the histogram filled with a yellow shade represents the distribution including the constraint from the measured microlens-parallax parameters, while the histogram shaded by slanted lines represents the distribution without the parallax constraint.

derived value of $\theta_{*}=0.56 \mu$ as is an upper limit and the additional uncertainty would propagate into $\theta_{\mathrm{E}, \min }$ and $\mu_{\min }$.

\section{Physical Lens Parameters}

As described by Equations (6) and (7) if both $\theta_{\mathrm{E}}$ and $\pi_{\mathrm{E}}$ are measured, then the mass $M$ and distance $D_{\mathrm{L}}$ can be uniquely determined. In the present case, $\pi_{\mathrm{E}}$ is measured but for $\theta_{\mathrm{E}}$ we have only a lower limit $\theta_{\mathrm{E}, \mathrm{min}}$, which is given by Equation (8). Therefore, we constrain the physical lens parameters by conducting a Bayesian analysis based on the constraints of the measured event timescale $t_{\mathrm{E}}$ and the microlens parallax $\pi_{\mathrm{E}}$ combined with $\theta_{\mathrm{E}, \mathrm{min}}$.

For the Bayesian analysis, one needs prior models of the mass function of lens objects and the density and dynamical distributions of Galactic matter. The source is a disk star and thus we need models to describe disk self-lensing events in which disk source stars are lensed by disk lenses. For the mass function, we employ Chabrier (2003). For the density distribution, we adopt the Han \& Gould (2003) model in which the disk is described by a double-exponential disk. For the velocity distribution, we use the dynamical model of Han \& Gould (1995), in which the motion of disk objects is modeled by a Gaussian about the disk rotation speed. OGLE-2017-BLG0482 is likely to be a disk self-lensing event, where a disk star is lensed by a foreground disk star. In the Bayesian analysis, we therefore locate both the source and lens following the disk matter distribution model. The top panel of Figure 8 shows the distribution $D_{\mathrm{S}}$. The range of the source distance as measured by $1 \sigma$ uncertainty is $D_{\mathrm{S}}=8.1_{-1.8}^{+1.6} \mathrm{kpc}$. We note that the presented $D_{\mathrm{S}}$ distribution is different from the distribution of disk stars because the lensing probability is higher for distant stars.

In the Bayesian analysis, we produce a large number of events by conducting a Monte Carlo simulation based on the prior models of the mass function, physical and dynamical distributions. We estimate $M$ and $D_{\mathrm{L}}$ and their uncertainties from the distributions of events with timescales and microlensparallax values located within the ranges of the measured values. In this process, we also impose the constraint of the lower limit of the angular Einstein radius, $\theta_{\mathrm{E}, \mathrm{min}}$, given in Equation (8), and the measured microlens parallax. We impose the microlens-parallax constraint by computing the covariance matrix based on the MCMC chain in order to consider the uncertainties of the north and east components of $\pi_{\mathrm{E}}$ and the orientation of the distributions in the $\pi_{\mathrm{E}, E}-\pi_{\mathrm{E}, N}$ plane.

In Figure 8, we present the distributions of the relative lenssource proper motion $\mu$, the angular Einstein radius $\theta_{\mathrm{E}}$, and the relative lens-source parallax $\pi_{\text {rel }}$ produced from the Bayesian analysis using the adopted Galactic models. We present two sets of distributions: one with (histogram filled with a yellow 
Table 2

Super-Earth Microlensing Planets with M-dwarf Hosts

\begin{tabular}{llll}
\hline \hline \multicolumn{1}{c}{ Planet } & Planet Mass & Host Mass & Reference \\
\hline OGLE-2005-BLG-390Lb & $5.5_{-2.7}^{+5.5} M_{\oplus}$ & $0.22_{-0.11}^{+0.21} M_{\odot}$ & Beaulieu et al. (2006) \\
MOA-2007-BLG-192Lb & $3.2_{-1.8}^{+5.2} M_{\oplus}$ & $0.084_{-0.012}^{+0.015} M_{\odot}$ & Bennett et al. (2008), Kubas et al. (2012) \\
MOA-2009-BLG-266Lb & $10.4 \pm 1.7 M_{\oplus}$ & $0.56 \pm 0.09 M_{\odot}$ & Muraki et al. (2011) \\
MOA-2010-BLG-328Lb & $9.2 \pm 2.2 M_{\oplus}$ & $0.11 \pm 0.01 M_{\odot}$ & Furusawa et al. (2013) \\
OGLE-2013-BLG-0341Lb & $2 M_{\oplus}$ & $0.13 M_{\odot}$ & Gould et al. (2014) \\
OGLE-2016-BLG-1195Lb & $1.43_{-0.32}^{+0.45} M_{\oplus}$ & $0.078_{-0.012}^{+0.016} M_{\odot}$ & Shvartzvald et al. (2017) \\
MOA-2012-BLG-505Lb & $6.7_{-3.6}^{+10.7} M_{\oplus}$ & $0.10_{-0.05}^{+0.16} M_{\odot}$ & Nagakane et al. (2017) \\
OGLE-2017-BLG-0482Lb & $9.0_{-4.5}^{+9.0} M_{\oplus}$ & $0.20_{-0.10}^{+0.20} M_{\odot}$ & This paper \\
\hline
\end{tabular}

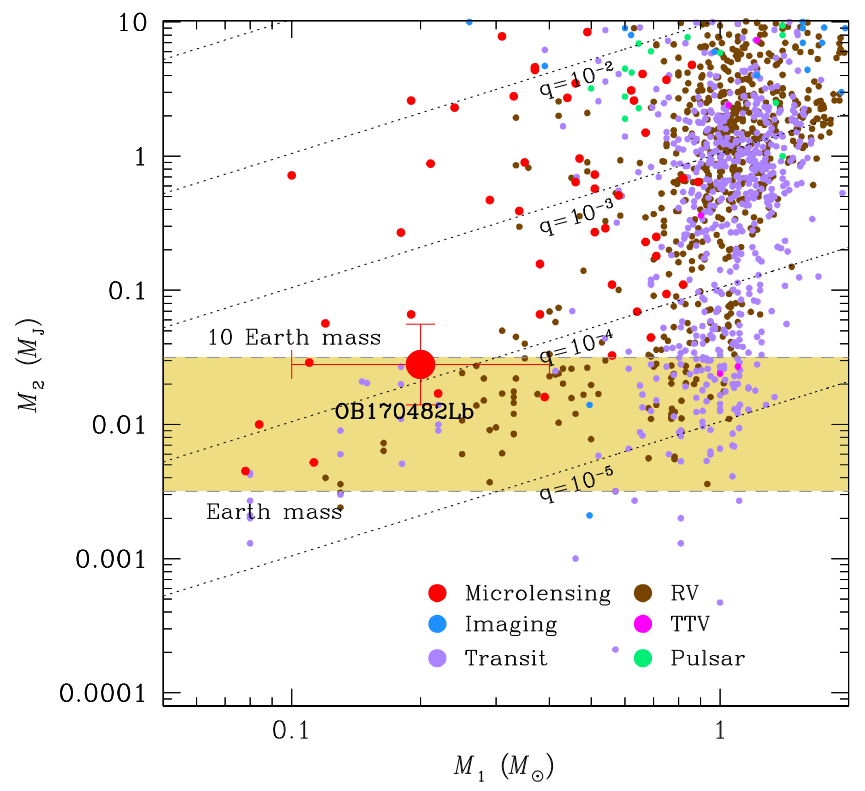

Figure 10. Distributions of planets with known masses in the parameter space of the host mass $M_{1}$ and the planet mass $M_{2}$. The position of OGLE-2017BLG-0482L is marked by a big red dot. The shaded area indicates the region of super-Earths for which the best estimates of the planet masses are in the range $1 M_{\oplus}<M_{2}<10 M_{\oplus}$. The dotted lines represent the mass ratios. The acronyms "RV" and "TTV" represent the radial-velocity and the transit-time-variation methods, respectively.

shade) and the other without (histogram shaded by slanted lines) the constraint of $t_{\mathrm{E}}$ and $\pi_{\mathrm{E}}$. From the comparison of the two sets of distributions, it is found that the constraint of $t_{\mathrm{E}}$ and $\pi_{\mathrm{E}}$ on the distributions is weak. There exist several reasons for this. First, the source did not cross the caustic and thus its relative size to the angular Einstein radius, $\rho$, cannot be constrained, preventing from measuring $\theta_{\mathrm{E}}=\theta_{*} / \rho$ and the relative proper motion between the source and the lens, $\mu=\theta_{\mathrm{E}} / t_{\mathrm{E}}$. Second, the upper limit given in Equation (8) is not a strong constraint because typical values of $\theta_{\mathrm{E}}$ are larger than $\sim 0.1$ mas. Finally, the constraint on the microlens parallax $\pi_{\mathrm{E}}$ is weak, as only one projection, i.e., $\pi_{\mathrm{E}, E}$, is constrained but the other one, i.e., $\pi_{\mathrm{E}, N}$, is poorly determined.

Figure 9 shows the distributions of the mass (upper panel) and the distance to the lens (lower panel) obtained from the Bayesian analysis. In each panel, we present two distributions. The histogram filled with a yellow shade represents the distribution obtained with the constraint of the event timescale and the microlens-parallax parameters, while the histogram shaded by slanted lines represents the distribution without the microlens-parallax constraint. From the comparison of the two distributions, it is found that the measured microlens-parallax parameters enable us to exclude lenses with very low masses located at small distances. On the other hand, we find that the constraint of $\theta_{\mathrm{E}, \min }$ on the physical lens parameters is too weak to significantly impact the posterior distribution.

It is found that the masses of the planet and the host are

$$
M_{\mathrm{p}}=9.0_{-4.5}^{+9.0} M_{\oplus}
$$

and

$$
M_{\text {host }}=0.20_{-0.10}^{+0.20} M_{\odot},
$$

respectively. We note that the masses and their uncertainties are estimated as the median values and the standard deviations of the distributions obtained from the Bayesian analysis. The mass of the planet is less than $10 M_{\oplus}$ and thus the planet is a superEarth according to the definition of Valencia et al. (2007). The mass of the host corresponds to that of late M-type dwarf. Therefore, the lens is a planetary system composed of a superEarth and a low-mass M-dwarf host.

The lens is located at a distance from Earth of

$$
D_{\mathrm{L}}=5.8_{-2.1}^{+1.8} \mathrm{kpc} \text {. }
$$

The projected separation of the planet from the host is

$$
a_{\perp}=1.8_{-0.7}^{+0.6} \mathrm{au} .
$$

We note that the physical lens parameters corresponding to the $u_{0}<0$ solution are similar to the presented values due to the similarity of the lensing parameters between the two degenerate solutions except the sign of $\pi_{\mathrm{E}, N}$.

\section{Discussion}

It is found that the planetary system OGLE-2017-BLG0482L is composed of a super-Earth orbiting a low-mass host star. Due to the faintness of host stars, such planetary systems are difficult to detect using other major planet detection methods such as the radial-velocity (RV) and transit methods in which planets are indirectly found from observations of host stars.

To demonstrate the high efficiency of the microlensing method to these planetary systems, in Figure 10, we present the distribution of planetary systems with known masses in the plane of the host mass $M_{1}$ and the planet mass $M_{2}$. We mark the location of OGLE-2017-BLG-0482L by a big red dot. In the plot, the shaded area indicates the region of super-Earths where the planet masses are in the range $1 M_{\oplus}<M_{2}<10 M_{\oplus}$. This shows that the microlensing method is sensitive to planets with low-mass hosts while the RV and transit methods are sensitive to planets orbiting solar-type stars. One also finds that 
the fraction of microlensing planets is especially high in the region of super-Earths with very low-mass $\left(M_{1} \lesssim 0.2 M_{\odot}\right)$ hosts. In Table 2, we list the super-Earth planets orbiting M-dwarf hosts detected using the microlensing method.

\section{Conclusion}

We analyzed the microlensing event OGLE-2017-BLG-0482, in which the light curve exhibited a short-term anomaly to the smooth lensing light curve. Analysis of the observed light curve indicated that the lens was a planetary system with a planet/host mass ratio of $q \sim 1.4 \times 10^{-4}$. We measured the microlens parallax $\pi_{\mathrm{E}}$ from the long-term deviation in the observed lensing light curve, but the angular Einstein radius $\theta_{\mathrm{E}}$ could not be measured. Using the measured $t_{\mathrm{E}}$ and $\pi_{\mathrm{E}}$, we found that the planetary system was composed of a super-Earth and a late M-dwarf host. The discovery of the planetary system demonstrates that microlensing provides an important tool to detect such planetary systems that are difficult to detect by other methods.

Work by C.H. was supported by the grant (2017R1A4A1015178) of National Research Foundation of Korea. The MOA project is supported by JSPS KAKENHI grant Nos. JSPS24253004, JSPS26247023, JSPS23340064, JSPS15H00781, and JP16H06287. The OGLE project has received funding from the National Science Centre, Poland, grant MAESTRO 2014/14/A/ST9/00121 to A.U. Work by A.G. was supported by JPL grant 1500811 . Work by J.C.Y. was performed under contract with the California Institute of Technology (Caltech)/Jet Propulsion Laboratory (JPL) funded by NASA through the Sagan Fellowship Program executed by the NASA Exoplanet Science Institute. Work by Y.S. was supported by an appointment to the NASA Postdoctoral Program at the Jet Propulsion Laboratory, California Institute of Technology, administered by Universities Space Research Association through a contract with NASA. We acknowledge the high-speed internet service (KREONET) provided by Korea Institute of Science and Technology Information (KISTI). This research has made use of the KMTNet system operated by the Korea Astronomy and Space Science Institute (KASI), and the data were obtained at three host sites of CTIO in Chile, SAAO in South Africa, and SSO in Australia.

\section{ORCID iDs}

C. Han (iD https://orcid.org/0000-0002-2641-9964

V. Bozza (ib https://orcid.org/0000-0003-4590-0136

D. P. Bennett (iD https://orcid.org/0000-0001-8043-8413

A. Fukui (iD https://orcid.org/0000-0002-4909-5763

Y. Itow (ii) https://orcid.org/0000-0002-8198-1968
N. Koshimoto (iD https://orcid.org/0000-0003-2302-9562

S. Miyazaki if https://orcid.org/0000-0002-1962-904X

N. Rattenbury (i) https://orcid.org/0000-0001-5069-319X

D. Suzuki (1) https://orcid.org/0000-0002-5843-9433

P. Pietrukowicz (1) https://orcid.org/0000-0002-2339-5899

J. Skowron (1D https://orcid.org/0000-0002-2335-1730

W.-T. Kim (i) https://orcid.org/0000-0003-4625-229X

Y. Shvartzvald (iD https://orcid.org/0000-0003-1525-5041

\section{References}

Alard, C., \& Lupton, R. H. 1998, ApJ, 503, 325

Albrow, M. D., Beaulieu, J.-P., Caldwell, J. A. R., et al. 2000, ApJ, 534, 894

Batista, V., Gould, A., Dieters, S., et al. 2011, A\&A, 529, 102

Beaulieu, J.-P., Bennett, D. P., Fouqué, P., et al. 2006, Natur, 439, 437

Bennett, D. P., Bond, I. A., Udalski, A., et al. 2008, ApJ, 684, 663

Bennett, D. P., \& Rhie, S. H. 1996, ApJ, 472, 660

Bensby, T., Yee, J. C., Feltzing, S., et al. 2013, A\&A, 549, A147

Bessell, M. S., \& Brett, J. M. 1988, PASP, 100, 1134

Bond, I. A., Abe, F., Dodd, R. J., et al. 2001, MNRAS, 327, 868

Bond, I. A., Bennett, D. P., Sumi, T., et al. 2017, MNRAS, 469, 2434

Boss, A. P. 1989, PASP, 101, 767

Chabrier, G. 2003, ApJL, 586, L133

Furusawa, K., Udalski, A., Sumi, T., et al. 2013, ApJ, 779, 91

Gaudi, B. S. 1998, ApJ, 506, 533

Gaudi, B. S. 2012, ARA\&A, 50, 411

Gould, A. 1992, ApJ, 392, 442

Gould, A., \& Loeb, A. 1992, ApJ, 396, 104

Gould, A., Udalski, A., Shin, I.-G., et al. 2014, Sci, 345, 46

Han, C. 2006, ApJ, 638, 1080

Han, C., \& Gould, A. 1995, ApJ, 447, 53

Han, C., \& Gould, A. 2003, ApJ, 592, 172

Han, C., \& Jeong, Y. 1998, MNRAS, 301, 231

Han, C., Jung, Y. K., Udalski, A., et al. 2013, ApJ, 778, 38

Hwang, K.-H., Choi, J.-Y., Bond, I. A., et al. 2013, ApJ, 778, 55

Hwang, K.-H., Udalsk, A., Bond, I. A., et al. 2017, arXiv:1711.09651

Kennedy, G. M., Kenyon, S. J., \& Bromley, B. C. 2006, ApJL, 650, L139

Kervella, P., Thévenin, F., Di Folco, E., \& Ségransan, D. 2004, A\&A, 426, 297

Kim, D.-J., Kim, H.-W., Hwang, K.-H., et al. 2018a, AJ, 155, 76

Kim, H.-W., Hwang, K.-H., Kim, D.-J., et al. 2018b, AJ, 155, 186

Kim, S.-L., Lee, C.-U., Park, B.-G., et al. 2016, JKAS, 49, 37

Kubas, D., Beaulieu, J. P., Bennett, D. P., et al. 2012, A\&A, 540, 78

Mao, S., \& Paczyński, B. 1991, ApJL, 374, L37

Muraki, Y., Han, C., Bennett, D. P., et al. 2011, ApJ, 741, 22

Nagakane, M., Sumi, T., Koshimoto, N., et al. 2017, AJ, 154, 35

Nataf, D. M., Gould, A., Fouqué, P., et al. 2013, ApJ, 769, 88

Schechter, P. L., Mateo, M., \& Saha, A. 1993, PASP, 105, 1342

Shvartzvald, Y., Yee, J. C., Calchi Novati, S., et al. 2017, ApJL, 840, L3

Skowron, J., Udalski, A., Gould, A., et al. 2011, ApJ, 738, 87

Smith, M. C., Mao, S., \& Paczyński, B. 2003, MNRAS, 339, 925

Sumi, T., Abe, F., Bond, I. A., et al. 2003, ApJ, 591, 204

Udalski, A. 2003, AcA, 53, 291

Udalski, A., Szymański, M. K., \& Szymański, G. 2015, AcA, 65, 1

Valencia, D., Sasselov, D. D., \& O'Connell, R. J. 2007, ApJ, 656, 545

Woźniak, P. R. 2000, AcA, 50, 421

Yee, J. C., Shvartzvald, Y., Gal-Yam, A., et al. 2012, ApJ, 755, 102

Yoo, J., DePoy, D. L., Gal-Yam, A., et al. 2004, ApJ, 603, 139 